\title{
Proinsulin Promotes Self-Renewal of a Hematopoietic Progenitor Cell Line In Vitro
}

\author{
Yuewen Han, ${ }^{1,2,3}$ Tingting Liu, ${ }^{1}$ Yunding Zou, ${ }^{1,4}$ Ling Ji, ${ }^{1}$ Yuanyuan Li, ${ }^{5}$ Jing Li, ${ }^{1}$ Jing Wang, \\ Guopin Chen, ${ }^{2}$ Jieping Chen, ${ }^{4}$ Liang Chen, ${ }^{6}$ and Zhijia Ye ${ }^{1,4}$
}

\author{
${ }^{1}$ Institute of Tropical Medicine, Third Military Medical University, Chongqing, China \\ ${ }^{2}$ Bioengineering College, Chongqing University, Chongqing, China \\ ${ }^{3} X i$ 'an Center for Disease Control and Prevention, Xi'an, China \\ ${ }^{4}$ Department of Hematology, Southwest Hospital, Chongqing, China \\ ${ }^{5}$ Biomedical Analysis Center, Third Military Medical University, Chongqing, China \\ ${ }^{6}$ Department of Plastic and Aesthetic Surgery, Southwest Hospital, Chongqing, China
}

Correspondence should be addressed to Zhijia Ye; zjye@tmmu.edu.cn

Received 8 January 2017; Accepted 19 April 2017; Published 3 July 2017

Academic Editor: Marco Songini

Copyright (C) 2017 Yuewen Han et al. This is an open access article distributed under the Creative Commons Attribution License, which permits unrestricted use, distribution, and reproduction in any medium, provided the original work is properly cited.

\begin{abstract}
The objective of this study was to assess the effects of exogenously expressed proinsulin on the biological characters of a hematopoietic stem cell line (HSC) and erythroid myeloid lymphoid (EML) cells and explore new strategies for cell therapy for type I diabetes. EML cells were transduced with lentivirus particles carrying the human proinsulin (proINS) gene. The positive transduced cells were selected based on green fluorescence protein (GFP) positivity and puromycin resistance. Overexpression of proINS was confirmed via real-time PCR and Western blotting. The functional activity of the human proINS secreted by EML cells was elucidated by analyzing the activation of insulin receptor and its downstream signaling. Pro-INS + EML cells were able to prime the phosphorylation of insulin receptor as well as induce the expression of downstream genes of insulin receptor. Furthermore, Wnt3a can significantly promote self-renewal of Pro-INS + EML cells. However, we did not observe significant changes in the proliferation and differentiation of INS + EML cells, compared to the control EML cells. Our results might be useful for developing a new therapy for diabetes mellitus.
\end{abstract}

\section{Introduction}

Diabetes mellitus, a widespread metabolic disorder, is a global epidemic with a continuous, steep increase in global prevalence and incidence [1]. There are two forms of diabetes mellitus: type I, when the pancreas does not produce enough insulin [2], and type II, when the body cannot effectively use the produced insulin [3]. In both cases, chronic high blood glucose levels cause severe complications, including macroangiopathy and microangiopathy, leading to blindness, chronic renal insufficiency, central and peripheral neuropathy, and cardiovascular disease [3].

Conventional therapy for diabetes based on exogenous insulin or oral agents may control and delay, but not prevent, disease-related complications $[2,4]$. Despite the promising outcomes observed with islet transplantation and progress in immunomodulatory therapies, the need for an effective cell replacement strategy for diabetes remains. Especially, stem cell-based strategies represent significant therapeutic potential owing to both the intrinsic regenerative capacity and the immunomodulatory potential of stem cells [5]. In the past years, substitution of failed $\beta$-cells by embryonic stem cells (ESCs) or induced pluripotent stem cells (iPSCs) raised hope for diabetes cure $[5,6]$. Some successful cases showed that procedures that promote ESC differentiation into insulin-secreting cells have been developed for animal models [5]. However, the risk of immune rejection and lack of efficacy in the conversion of ESCs into $\beta$-cells are yet to be tackled. As iPSCs are generated from autologous cells, graft-directed immune destruction is likely circumvented by 
reprogramming the patient's specific fibroblast into type 1 diabetes patient-specific iPSCs [7]; however, generating human iPSCs suitable for clinical application is still a long way ahead. In addition, formation of $\beta$-cells from ESCs as well as iPSCs is technically problematic and quite expensive [5].

Hematopoietic stem cells (HSCs) are multipotent stem cells residing in the bone marrow and umbilical cord blood, capable of differentiating into different lineages of blood cells [8]. More interestingly, HSCs have the potential to correct and/or reeducate the immune aberration that promotes autoimmune attacks against host tissue. Therefore, HSC transplantation has been used as a therapeutic strategy for different types of autoimmune diseases and applied to type 1 diabetes patients in a minimum of two clinical trials with promising results $[9,10]$. Previous studies show that insulin can be an important factor for HSC modulation. Interactions between the insulin signaling pathway and the canonical Wnt signaling pathway can promote relatively stable self-renewal of HSCs [11, 12]. For example, insulin activates PI3K/Akt pathway, inhibits apoptosis of HSCs, and promotes expansion of HSCs. Combined treatment with insulin and CHIR99021, a GSK inhibitor, can support efficient HSC expansion in vitro [12]. However, whether and how the exogenously expressed proinsulin affects the biological characteristics of HSCs remains unknown. In this study, we report the effects of exogenous proinsulin on the self-renewal and differentiation of EML cells, which had been demonstrated to be a unique model very similar to wild-type HSCs [13].

\section{Materials and Methods}

2.1. Cell Lines and Culture. Erythroid myeloid lymphoid (EML) cells were cultured with Iscove's modified Dulbecco's medium (IMDM), supplemented with $10 \%$ fetal bovine serum (FBS) (Hyclone, Logan, UT, USA), 15\% conditional medium of BHK-21 cells (containing stem cell factor), $100 \mathrm{U} / \mathrm{ml}$ penicillin, and $100 \mu \mathrm{g} / \mathrm{ml}$ streptomycin. To construct a proinsulin (Pro-INS) overexpressing cell line, the human Pro-INS open reading frame (GenBank number, NM_000207) was synthesized and recombined into the lentivirus vector pCDH-CMV-MCS-EF1-GFP-T2A-Puro. The pCDH-Pro-INS or pCDH plasmids were cotransfected with the packaging plasmids (psPAX2 and pMG2.D) into 293FT cells (around $70-80 \%$ confluency). The medium was changed after $12 \mathrm{~h}$, and the virus-containing supernatant was harvested $48 \mathrm{~h}$ later. The resulting virus-containing supernatant was then used to infect EML cells, and the positive cells were screened using the dual markers for GFP and puromycin.

\subsection{Quantitative Real-Time Reverse-Transcription Polymerase} Chain Reaction (RT-qPCR). Total RNA was isolated from the cultured cells using the RNeasy Mini Kit (QIAGEN) as per the manufacturer's protocol. Total RNA was reverse-transcribed using the M-MLV RTase cDNA Synthesis Kit (TaKaRa). Real-time quantitative RT-PCR was performed with the iQ SYBR Green Supermix (Bio-Rad) and normalized against GAPDH expression. The primers are as follows: insulin, $5^{\prime}$ CAGCCGCAGCCTTTGT- $3^{\prime}$ and 5'-TTCCACAATGCCA CGCT-3'; insulin receptor (IR), 5'-CGAGTGCCCGTCTG
GCTATA-3' and 5'-GGCAGGGTCCCAGACATG-3'; GLU T1, 5'-GAGCATCTTCGAGAAGGCAGGTGT- $3^{\prime}$ and $5^{\prime}$ GGCCACAATGAACCATGGAATA-3'; and GAPDH, 5'TGTGTCCGTCGTGGATCTGA- $3^{\prime}$ and $5^{\prime}$-CCTGCTTCAC CACCTTCTTGAT-3'.

2.3. Western Blotting. For Western blotting, the cells were collected and lysed in radioimmunoprecipitation assay (RIPA) buffer with protease inhibitors on ice. Approximately $30 \mu \mathrm{g}$ of the sample was boiled for $5 \mathrm{~min}$, separated using $10 \%$ SDS-PAGE, and then transferred to polyvinylidene fluoride (PVDF) membranes (Millipore, Billerica, MA, USA). After incubation with a blocking buffer, the membranes were incubated with mouse anti-insulin (Cell Signaling Technology, Danvers, MA, USA), mouse anti-insulin receptor $\beta$ (Cell Signaling), rabbit anti-phospho-IGF-I receptor $\beta$ (Tyr1131) (Cell Signaling), mouse anti-phospho-Akt (Thr308) (Abcam), and rabbit anti- $\beta$-actin (Cell Signaling) primary antibodies and horseradish peroxidase-conjugated secondary antibodies, including horse anti-mouse and goat anti-rabbit antibodies (Cell Signaling).

2.4. Flow Cytometry. Cells were harvested, and single-cell suspensions were prepared in cool phosphate-buffered saline (PBS) containing $0.5 \%$ bovine serum albumin (BSA) and stained with fluorescein isothiocyanate (FITC) anti-CD34 (BD Biosciences, California, USA), FITC Annexin V (BioLegend, CA, USA), allophycocyanin (APC) anti-B220 (eBioscience, CA, USA), phycoerythrin (PE) anti-Sca-1 (eBioscience), and PE-CD11b (eBioscience). All samples were analyzed using FACS Aria I (BD Biosciences), and the data were analyzed using the FlowJo software (Tree Star Inc.).

2.5. 5-Bromo-2'-deoxyuridine (Brdu) Incorporation Assay. The cells were pulsed with $20 \mu \mathrm{M}$ BrdU, maintained in the medium for $30 \mathrm{~min}$, and collected and fixed in cold $70 \%$ ethanol. The fixed cells were treated with $4 \mathrm{~N} \mathrm{HCl}$, neutralized with $0.1 \mathrm{M}$ borax, and washed in PBS containing $0.05 \%$ BSA. Next, the cells were incubated with anti-BrdU antibody (BD Biosciences) in $0.5 \%$ BSA, followed by incubation with FITC-conjugated anti-mouse secondary antibody (Sigma) in PBS with $0.5 \%$ Tween-20. The cells were then resuspended in PBS containing propidium iodide (PI) and RNase A and analyzed via fluorescence-activated cell sorting (FACS).

2.6. CFU-Based Assay. To assess their differentiation capacity, total EML/control and EML/proINS cells were plated in $1 \mathrm{ml}$ of MethoCult H4035 Optimum without EPO (Stemcell Technologies, Vancouver, Canada) in 6 -well plates $\left(10^{3}\right.$ cells/well) in replicates. The medium was supplemented with $8 \mathrm{U} / \mathrm{ml}$ recombinant human erythropoietin (PeproTech, NJ, USA) for the generation of erythroid burst-forming units (BFU-E). The cultures were maintained at $37^{\circ} \mathrm{C}, 5 \% \mathrm{CO}_{2}$ for 10-14 days. The BFU-E, CFU-GM, and CFU-Meg colonies were counted after 10-14 days of plating.

\section{Results}

3.1. Exogenous Expression of Proinsulin in EML Cells. To construct stable EML cell lines expressing proinsulin, we cloned 


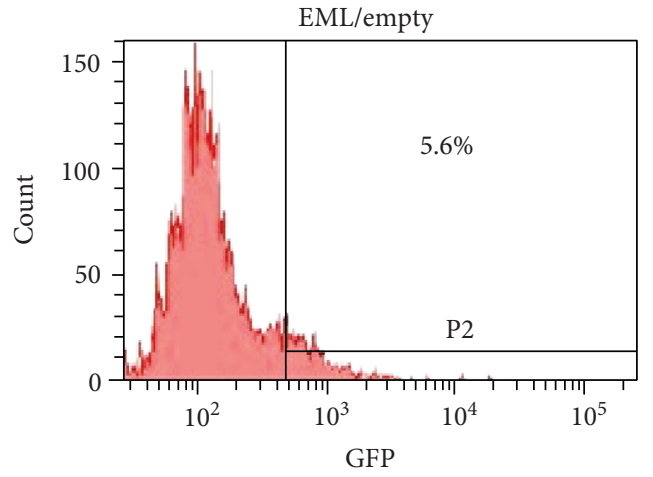

(a)

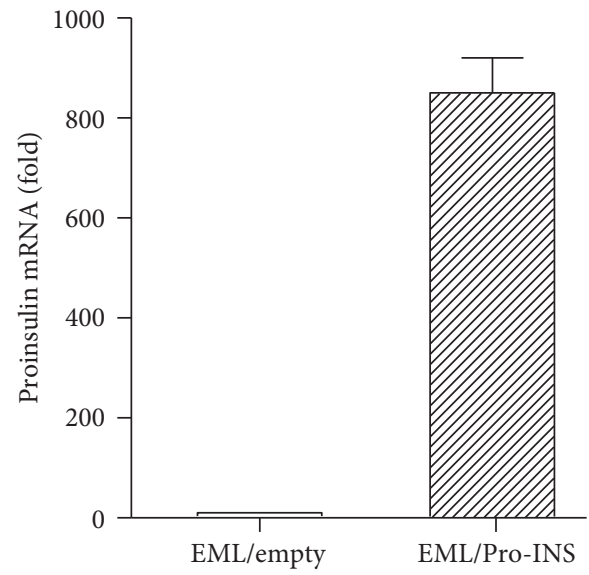

(c)

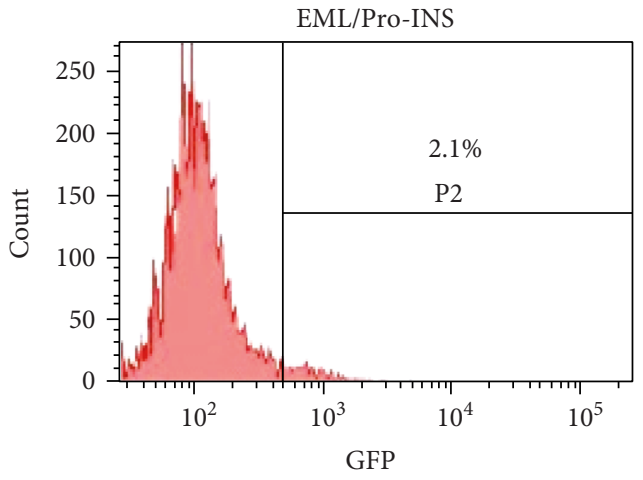

(b)

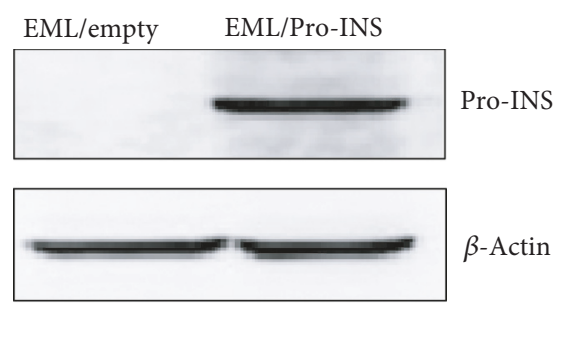

(d)

FIgURE 1: Overexpressed proinsulin identified in EML cells. (a), (b) GFP-positive cells were detected via FACS in EML cells transduced by lentivirus particles carrying empty vector (empty) or proinsulin gene (Pro-INS), respectively. (c) Overexpressed proinsulin mRNA was detected using real-time PCR in EML cells transduced by LV-V carrying the proinsulin gene. (d) Proinsulin protein was detected via Western blotting in EML cells transduced by LV-V carrying the proinsulin gene.

INS cDNA into the lentivirus vector pCDH-CMV-MCSEF1-GFP-T2A-Puro, generated pseudoviral particles carrying INS, and transduced EML cells with the lentivirus particles at an ROI of $100: 1$. Because the lentivirus vector contains both GFP gene and puromycin resistance gene, we used both markers for evaluation and selection of transductants. On day 1 after infection, 5\% GFP-positive EML cells were detected (Figures 1(a) and 1(b)). EML cells with stable GFP expression were obtained on day 10 after puromycin selection. Unlike the control EML cells transduced with lentivirus particles carrying empty vector (EML/empty), EML cells transduced with lentivirus particles carrying the proinsulin gene (EML/Pro-INS) were detected based on the expression of proinsulin. INS mRNA and proinsulin protein were verified using real-time quantitative PCR (Figure 1(c)) and Western blotting (Figure 1(d)), respectively. Approximately $10 \mathrm{pg} / \mathrm{ml}$ proinsulin was detected in the supernatant of the culture medium of EML/Pro-INS cells via ELISA analysis (data not shown).

3.2. Secreted Proinsulin Activated the Insulin Receptor Pathway. Proinsulin is a prohormone with low metabolic activity compared to mature insulin. Proinsulin differentially binds to and activates the two insulin receptor (IR) isoforms, with higher affinity for IR-A than IR-B [14], and then the activated $\beta$-subunits of IR consequently activate the downstream molecules and turn on signaling pathways, including Src/PI3K/Akt [15]. To determine whether the proinsulin secreted by EML/Pro-INS can activate the IR-mediated signaling pathway, we analyzed the phosphorylation of $\beta$ subunits of IR and the phosphorylation of Akt in mouse embryonic fibroblast (MEF) cells stimulated with the CM of EML/Pro-INS. We found that the phosphorylation levels of IR and Akt significantly increased in the MEF cells treated with 20\% CM of EML/Pro-INS cells (Figure 2(a)). The phosphorylation levels of IR positively correlated with the doses of the added CM concentration (Figures 2(b) and 2(c)). We also assayed the gene expression levels of IR and Glut1, which are involved in the regulation of glucose metabolism, and found that the expression levels of IR and Glut1 significantly increased in MEF cells (Figure 2(d)).

3.3. Exogenously Expressed Proinsulin Had No Significant Influence on the Proliferation and Differentiation of Hematopoietic Progenitor Cells. Proinsulin inhibits apoptosis and promotes the survival of neuroepithelial cells or 


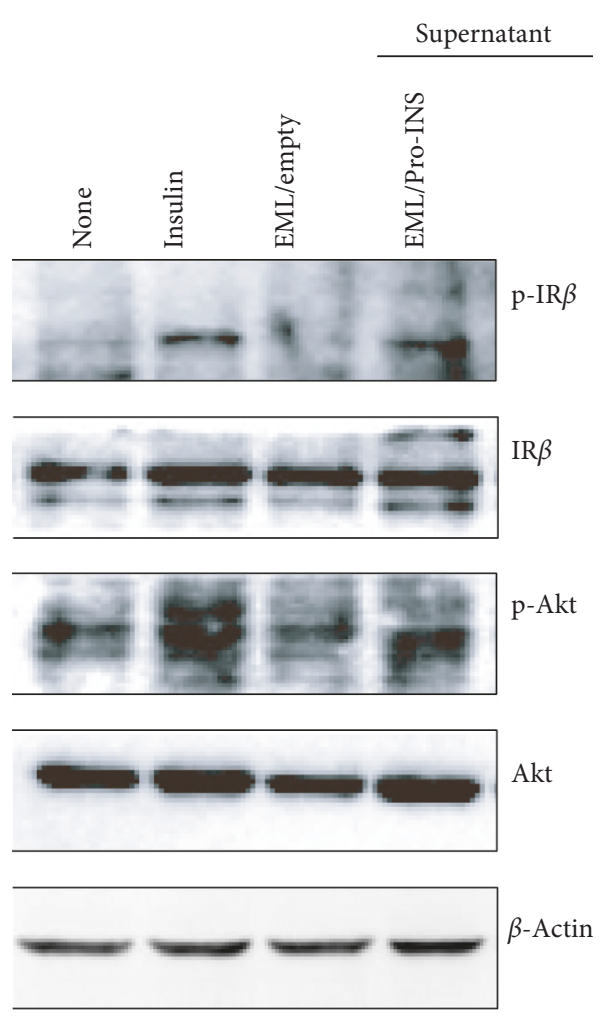

(a)

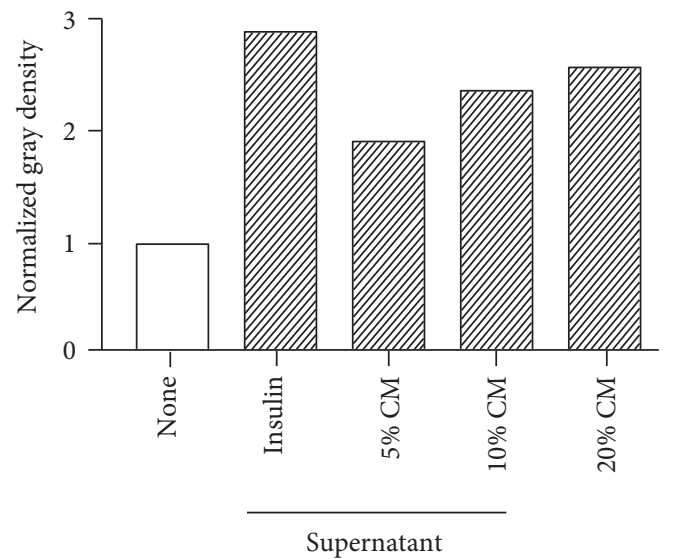

(c)

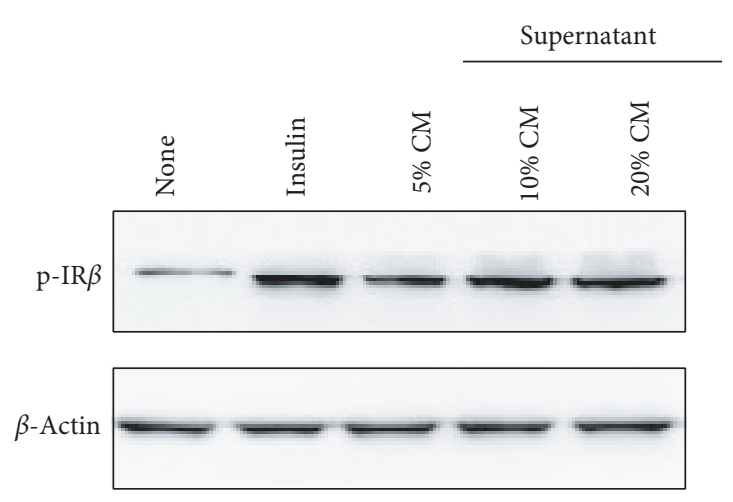

(b)

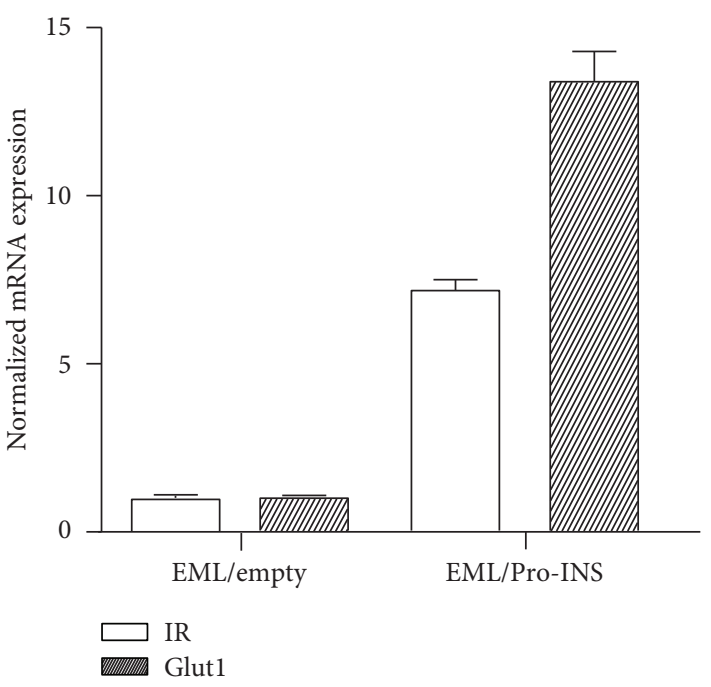

(d)

FIgURE 2: Signaling pathway mediated by the insulin receptor (IR) activated by proinsulin. (a) Serum-starved MEF cells (for $12 \mathrm{~h}$ ) were stimulated with insulin, 20\% CM of EML/empty, and 20\% CM of EML/Pro-INS, respectively, for 10 min. Then, the cells were harvested and lysed in RIPA buffer. The phosphorylated $\beta$-subunit of IR $(\operatorname{IR} \beta)$ and phosphorylated Akt were detected via Western blotting. (b) Serum-starved MEF cells (for $12 \mathrm{~h}$ ) were exposed to insulin for $10 \mathrm{~min}$ and increasing doses of CM of EML/Pro-INS, respectively. The phosphorylated IR $\beta$ was detected via Western blotting. The ratio of the gray density of phosphor-IR $\beta$ versus total IR $\beta$ was measured and calculated. (c), (d) The levels of IR and Glut1 mRNA expression in MEF cells stimulated with 20\% CM of EML/empty and 20\% CM of EML/Pro-INS, respectively, were detected using real-time PCR.

neurons during early neural development [16]. In order to assess whether proinsulin affects the proliferation of EML cells, we measured the proliferation capacity of EML/ProINS cells by Brdu incorporation assay. We found no significant difference in Brdu incorporation between EML/
Pro-INS cells and control cells (Table 1, Figure 3(a)). In contrast, the population of Annexin V-positive cells in EML/proINS is lesser than that in EML/control cells (Table 1). Thus, the expressed proinsulin prohibits the apoptosis of EML cells. 
TABle 1: Proliferation and apoptosis of EML/empty and EML/ Pro-INS.

\begin{tabular}{lcccc}
\hline & \multicolumn{2}{c}{ EML/empty } & \multicolumn{2}{c}{ EML/Pro-INS } \\
& Control & Wnt3a & Control & Wnt3a \\
\hline BrdU $+\%$ & $56.3 \pm 5.7$ & $65.5 \pm 5.9$ & $58.9 \pm 5.2$ & $68.8 \pm 6.4$ \\
Annexin V+\% & $15.5 \pm 1.8$ & $14.9 \pm 1.6$ & $11.9 \pm 1.2$ & $10.2 \pm 1.8$ \\
\hline
\end{tabular}

Previously, we found that $\mathrm{CD} 34^{+}$Sca-1hi EML cells autonomously differentiated into $\mathrm{CD} 34^{-} \mathrm{Sca}-1$ low after being cultured in SCF medium [17]. In this study, we addressed the two questions: whether and how the exogenously expressed proinsulin influences the distribution of $\mathrm{CD} 34^{+} \mathrm{Sca}-1 \mathrm{hi}$ and $\mathrm{CD} 34^{+} \mathrm{Sca}-1$ low subpopulations in EML cells. Interestingly, we did not observe a significant difference in the distribution of CD $34^{+}$Sca-1hi and CD34-Sca-1low in both EML/Pro-INS and EML/empty cells (Figure 3(b)). This indicated that proinsulin had no effect on self-renewal and differentiation of population of $\mathrm{CD}_{3} 4^{+} \mathrm{Sca}-1 \mathrm{hi}$.

EML cell line has a well-documented multilineage differentiation capacity and can differentiate into erythroid, myeloid, and lymphoid progenitors in the presence of SCF $[13,18]$. In this study, we studied the influence of expressed proinsulin on lineage differentiation by checking $\mathrm{CFU}$ formation. We observed very similar patterns of BFU-E and CFU-GM and CFU-Meg in both EML/ProINS cells and EML/empty cells (Figure 3(c)). These results indicated that there were no significant effects of the exogenously expressed proinsulin on the lineage-biased differentiation of EML cells. The data obtained for cell cycle profile assay involving PI staining showed that there were significantly different population sizes in the G0/G1 and S/G2/M phases in EML/proINS, compared to EML/ empty (Figure 3(d)). Cumulatively, exogenously expressed proinsulin did not affect the ability of proliferation and lineage differentiation of EML cells.

\subsection{Effects of the Interaction between Wnt3a and Proinsulin} on Self-Renewal and Differentiation of Hematopoietic Progenitor Cells. Wnt signaling is required for normal selfrenewal and other functions of HSCs. The synergistic effects of $\mathrm{Wnt} / \beta$-catenin signaling and PI3K/Akt signaling on the self-renewal and expansion of normal HSCs were observed in vitro and in vivo $[11,12]$. The combination of Wnt signaling activator (Wnt3a and CHIR99021) and insulin was shown to completely instead of the conventional cytokines and growth factors that are required for the expansion of normal HSCs in vitro. To verify whether proinsulins like insulin can cooperate with Wnt signaling to regulate self-renewal and differentiation of EML cells, we assayed the proliferation and differentiation of INS + EML cells treated by Wnt3a, the typical activator of Wnt signaling. We found that the significantly increased proliferation induced by Wnt3a was observed only in INS + EML cells, but not in the mock EML cells. Wnt3a not only decreased the population of Annexin V-positive INS + EML cells but also increased Brdu incorporation (Table 1). Those data indicated that the synergy of proinsulin and Wnt3a promoted the proliferation of EML cells by inhibiting cell apoptosis and enhancing cell growth (Figures 4(a) and 4(b)).

Similarly, we assayed the effects of Wnt3a on proliferative self-renewal and lineage differentiations of INS + EML cells. We found that the ratio of subpopulation of $\mathrm{CD} 34^{+}$Sca-1hi versus that of $\mathrm{CD} 34^{-}$Sca-1low increased on Wnt3a stimulation in EML/proINS compared to EML/ empty (Figure 4(c)). Meanwhile, we analyzed the lineage differentiation in EML/proINS by detecting lineage markers, including B220 and CD11b (Figure 4(c)). Wnt3a treatment did not have a significant effect on lineage differentiation in EML/Pro-INS compared to control EML cells. These results indicated that the combination of proinsulin and Wnt3a promoted self-renewal of the hematopoietic progenitors but did not inhibit their lineage differentiation.

\section{Discussion}

As a prohormone, proinsulin had been shown to have low metabolic activity compared to mature insulin. However, proinsulin can promote other effects in the regulation of growth and development in the embryonic stages [19-21]. Proinsulin is expressed in the developing chicken retina; it plays an autocrine/paracrine stimulatory role during neurogenesis and prevents cell death of embryos subjected to growth factor deprivation. Proinsulin was expressed in the rat yolk sac, where hematopoietic stem cells emerged in the late gestational stages [22]. The effects of proinsulin on the self-renewal and differentiation of HSCs remain unknown.

The EML cell line, generated by a transducing retrovirus carrying dominant mutated amino acid receptor, represents a good model for studying hematopoietic stem cells. EML cells are expanded from a single-cell clone, but contain heterogeneous subsets, displaying different phenotypes and functions. In our previous study, we isolated and identified two subpopulations, Lin-c-kit $+\mathrm{CD} 34^{+}$Sca- 1 hi and LinCD34 ${ }^{-}$Sca-1low, from parental EML cells. We found that the $\mathrm{CD} 34^{+}$Sca-1hi cells were able to undergo self-renewal and differentiate into $\mathrm{CD} 34^{-}$Sca-1low cells as well. We assumed that $\mathrm{CD} 34^{+}$cells represented the phenotype of the original EML clone. In this study, we evaluated the influence of exogenously expressed proinsulin on the proliferation of total EML cells and distribution of subpopulations. We found that the exogenous expression of proinsulin enhanced the proliferation of total EML by inhibiting apoptosis. On the other hand, we did not observe significant changes in the distribution of subpopulations. These results indicated that proinsulin can be a potentially useful reagent for promoting the proliferation of HSCs without influencing. This observation corresponds to some recent reports on the effect of matured insulin on mouse wild-type HSCs [11].

Wnt signaling has been demonstrated to play a significant role in regulating self-renewal and differentiation of HSCs [23]. Various interactions of Wnt signaling and other signaling pathways coordinate the expansion and differentiation of HSCs as well as the progenitors of different stages. Evidence supports the idea that mTOR signal pathway and Wnt signal pathway synergistically increased 


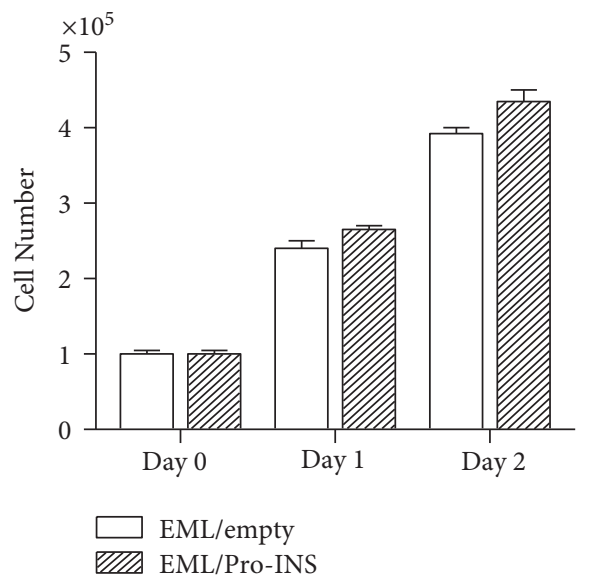

(a)

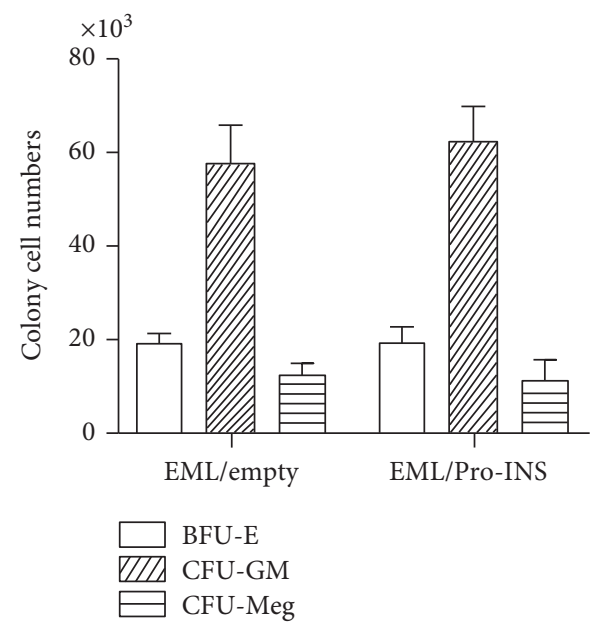

\begin{tabular}{lccc}
\hline & BFU-E & CFU-GM & CFU-Meg \\
\hline EML/empty & $18.9 \pm 2.2$ & $57.6 \pm 6.8$ & $12.3 \pm 2.3$ \\
EML/Pro-INS & $19.2 \pm 3.5$ & $60.2 \pm 6.8$ & $11.3 \pm 3.9$ \\
\hline
\end{tabular}

(c)

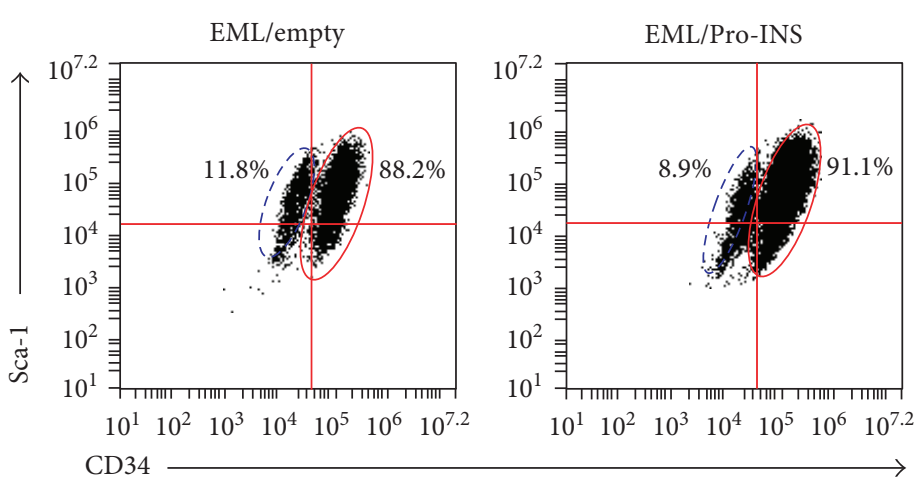

(b)

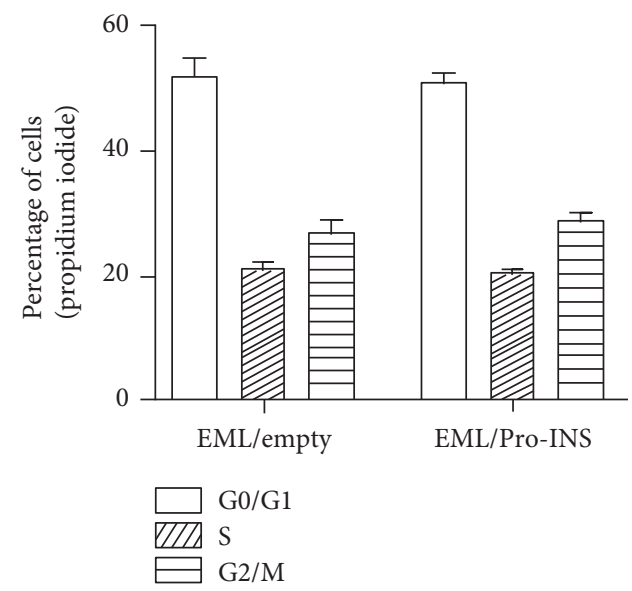

\begin{tabular}{lccc}
\hline & G0/G1 & $\mathrm{S}$ & $\mathrm{G} 2 / \mathrm{M}$ \\
\hline EML/empty & $51.6 \pm 3.3$ & $21.4 \pm 1.0$ & $27.1 \pm 2.9$ \\
EML/Pro-INS & $50.0 \pm 1.8$ & $21.2 \pm 0.8$ & $29.2 \pm 2.5$ \\
\hline
\end{tabular}

(d)

FIGURE 3: Effects of exogenous expression of proinsulin on the proliferation, self-renewal, and differentiation of EML cells. (a) EML/empty cells and EML/Pro-INS cells were cultured in fresh EML medium at an initial concentration of $1 \times 10^{5}$. Cell numbers were determined using trypan blue on days 1 and 2. (b) The distribution of the $\mathrm{CD} 34^{+} \mathrm{Sca}-1$ hi and $\mathrm{CD} 34^{-} \mathrm{Sca}-1$ low subpopulations in EML/empty cells and EML/Pro-INS cells was studied via FACS. (c) CFU-based assay of the differentiation capacity of EML/empty cells and EML/Pro-INS cells into erythroid, granulocyte-macrophage, and megakaryocytic lineages. (d) Analysis of the cell cycle profiles of EML/empty cells and EML/ Pro-INS cells by propidium iodide staining.

self-renewal of human cord blood HSCs and mouse bone marrow HSCs [12]. In this study, we observed a significant synergistic nature between the exogenously expressed proinsulin and Wnt3a stimulation on promoting the selfrenewal ability of $\mathrm{CD} 34^{+}$cells and repressing differentiation of $\mathrm{CD}_{3} 4^{+}$cells. Insulin activates $\mathrm{mTORC} 1 / 2$ and its downstream molecules via IRs and regulates the metabolism and proliferation of targeted cells. Malaguarnera et al. reported that proinsulin promoted the phosphorylation of p70S6K and Erk to an extent similar to insulin [14]. Furthermore, the group found that proinsulin played almost as equipotent roles as insulin in inducing cell proliferation and migration in cells expressing IR-A, although the metabolic activity was lower [14].
Proinsulin acts similar to insulin in reducing blood glucose [24]. Intravenous administration of adenovirus carrying proinsulin reversed high blood glucose levels in a mouse diabetes model [25]. In this study, we found that exogenously expressed proinsulin stimulated the phosphorylation of IR and induced the expression of Glut1, which mediates glucose uptake by the target cells. We observed that proinsulin induced similar levels of phosphorylation of IR than mature insulin did. The levels of phosphorylated IR induced by proinsulin positively correlated with the dose of proinsulin. On binding with IR, insulin activates several signaling pathways and induces expression of target genes, which are involved in the regulation of glucose metabolism. As one of the target genes of 


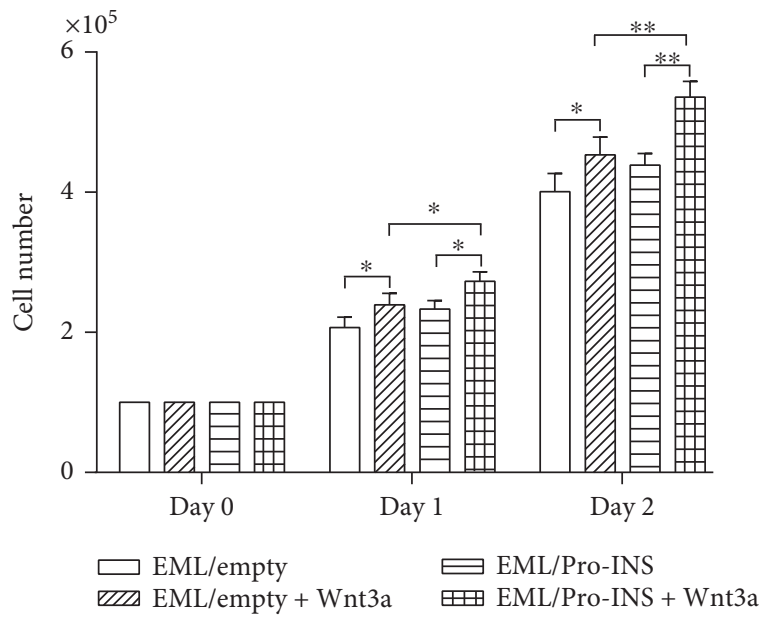

(a)

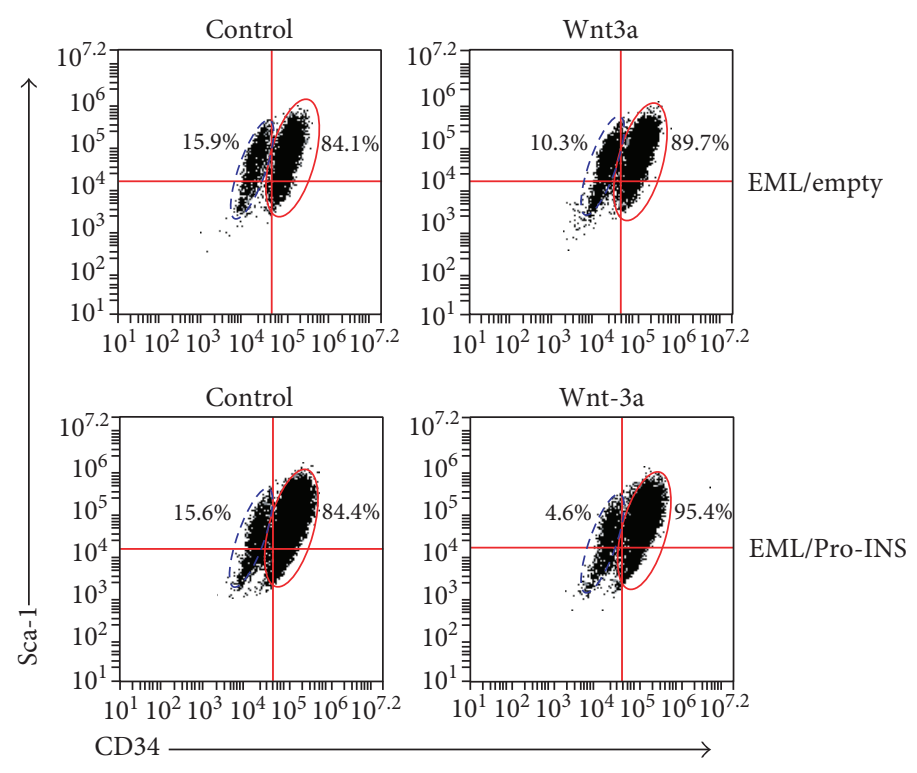

(b)

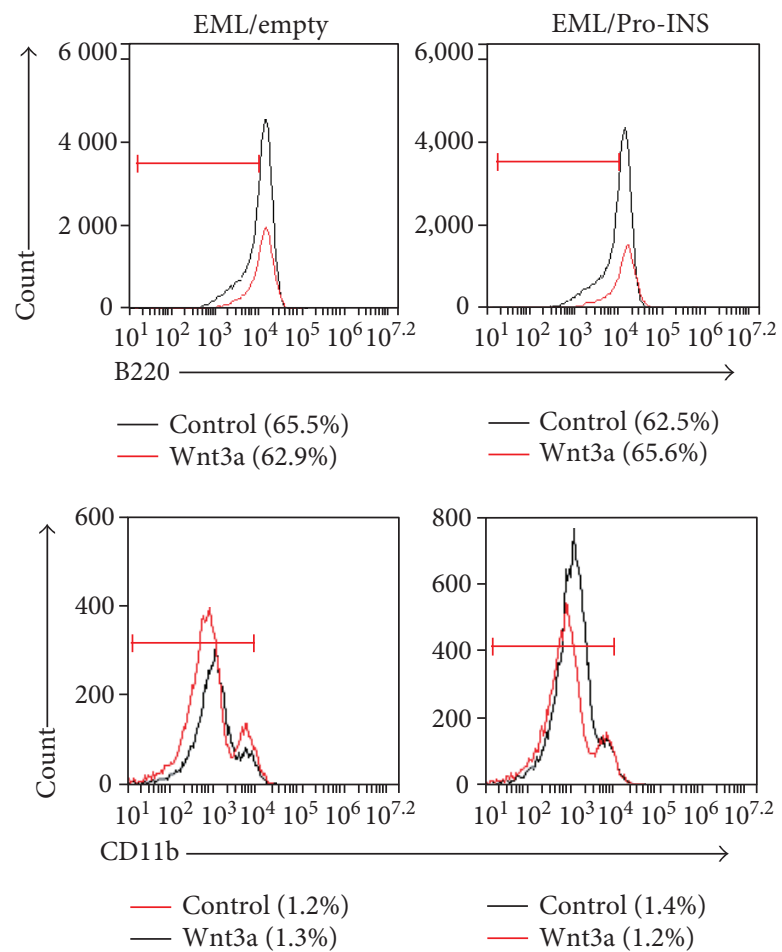

(c)

FIGURE 4: Synergistic effects of proinsulin and Wnt3a on the proliferation, self-renewal, and differentiation of EML cells. (a) EML/empty cells and EML/Pro-INS cells were cultured in fresh EML medium with or without Wnt $3 \mathrm{a}(50 \mathrm{ng} / \mathrm{ml})$ at an initial concentration of $1 \times 10^{5}($ day 0$)$. The total number of living cells assessed via trypan blue staining was counted on days 1 and 2 . (b) The distribution of CD34 ${ }^{+}$Sca- 1 hi and CD34- Sca-1low subpopulations was detected via FACS. (c) The percentage of lineage marks of B220 and CD11b was tested in the above cells via FACS. ${ }^{*} p<0.05$ and ${ }^{* *} p<0.001$.

the signaling pathways mediated by insulin, Glut1 plays a critical role in enhancing glucose uptake. Our results indicated that proinsulin, a kind of prohormone, is involved not only in regulating the growth and differentiation of HSCs but also in glucose metabolism. It is important to fully elucidate the physiological functions of the exogenously expressed proinsulin in regulating HSCs as well as its potential pharmacological application in diabetes therapy.

\section{Conflicts of Interest}

No competing financial interests exist. 


\section{Authors' Contributions}

Yuewen Han, Tingting Liu, and Yunding Zou contributed equally to this work.

\section{Acknowledgments}

The authors are grateful to Dr. PM, Rabinovich (Yale Medical School) for the discussion and critical reading of the manuscript. This research was supported by the National Natural Science Foundation of China (no. 81170471, no. 31371393) and the Natural Science Foundation of Chongqing Science and Technology Commission (no. cstc2013jjB10027, no. cstc2014yykfA110005).

\section{References}

[1] R. M. Bergenstal, A. J. Ahmann, T. Bailey et al., "Recommendations for standardizing glucose reporting and analysis to optimize clinical decision making in diabetes: the ambulatory glucose profile (AGP)," Diabetes Technology \& Therapeutics, vol. 15, no. 3, pp. 198-211, 2013.

[2] J. A. Bluestone, K. Herold, and G. Eisenbarth, "Genetics, pathogenesis and clinical interventions in type 1 diabetes," Nature, vol. 464, no. 7293, pp. 1293-1300, 2010.

[3] F. M. Ashcroft and P. Rorsman, "Diabetes mellitus and the beta cell: the last ten years," Cell, vol. 148, no. 6, pp. 1160-1171, 2012.

[4] M. A. Brehm, A. C. Powers, L. D. Shultz, and D. L. Greiner, "Advancing animal models of human type 1 diabetes by engraftment of functional human tissues in immunodeficient mice," Cold Spring Harbor Perspectives in Medicine, vol. 2, no. 5, article a007757, 2012.

[5] F. W. Pagliuca and D. A. Melton, "How to make a functional beta-cell," Development, vol. 140, no. 12, pp. 2472-2483, 2013.

[6] W. Li, M. Nakanishi, A. Zumsteg et al., "In vivo reprogramming of pancreatic acinar cells to three islet endocrine subtypes," eLife, vol. 3, no. 3, article e01846, 2014.

[7] R. Maehr, S. Chen, M. Snitow et al., "Generation of pluripotent stem cells from patients with type 1 diabetes," Proceedings of the National Academy of Sciences of the United States of America, vol. 106, no. 37, pp. 15768-15773, 2009.

[8] D. Bryder, D. J. Rossi, and I. L. Weissman, "Hematopoietic stem cells: the paradigmatic tissue-specific stem cell," The American Journal of Pathology, vol. 169, no. 2, pp. 338346, 2006.

[9] J. C. Voltarelli, C. E. Couri, A. B. Stracieri et al., "Autologous nonmyeloablative hematopoietic stem cell transplantation in newly diagnosed type 1 diabetes mellitus," JAMA, vol. 297, no. 14, pp. 1568-1576, 2007.

[10] E. Snarski, A. Milczarczyk, T. Torosian et al., "Independence of exogenous insulin following immunoablation and stem cell reconstitution in newly diagnosed diabetes type I," Bone Marrow Transplantation, vol. 46, no. 4, pp. 562-566, 2011.

[11] J. M. Perry, X. C. He, R. Sugimura et al., "Cooperation between both Wnt/\{beta\}-catenin and PTEN/PI3K/Akt signaling promotes primitive hematopoietic stem cell self-renewal and expansion," Genes \& Development, vol. 25, no. 18, pp. 19281942, 2011.

[12] J. Huang, M. Nguyen-McCarty, E. O. Hexner, G. DanetDesnoyers, and P. S. Klein, "Maintenance of hematopoietic stem cells through regulation of Wnt and mTOR pathways," Nature Medicine, vol. 18, no. 12, pp. 1778-1785, 2012.

[13] S. Tsai, S. Bartelmez, E. Sitnicka, and S. Collins, "Lymphohematopoietic progenitors immortalized by a retroviral vector harboring a dominant-negative retinoic acid receptor can recapitulate lymphoid, myeloid, and erythroid development," Genes \& Development, vol. 8, no. 23, pp. 2831-2841, 1994.

[14] R. Malaguarnera, A. Sacco, C. Voci, G. Pandini, R. Vigneri, and A. Belfiore, "Proinsulin binds with high affinity the insulin receptor isoform $\mathrm{A}$ and predominantly activates the mitogenic pathway," Endocrinology, vol. 153, no. 5, pp. 2152-2163, 2012.

[15] A. Sacco, A. Morcavallo, G. Pandini, R. Vigneri, and A. Belfiore, "Differential signaling activation by insulin and insulin-like growth factors I and II upon binding to insulin receptor isoform A,” Endocrinology, vol. 150, no. 8, pp. 3594-3602, 2009.

[16] E. J. de la Rosa and F. de Pablo, "Proinsulin: from hormonal precursor to neuroprotective factor," Frontiers in Molecular Neuroscience, vol. 4, no. 4, p. 20, 2011.

[17] Z. J. Ye, Y. Kluger, Z. Lian, and S. M. Weissman, "Two types of precursor cells in a multipotential hematopoietic cell line," Proceedings of the National Academy of Sciences of the United States of America, vol. 102, no. 51, pp. 18461-18466, 2005.

[18] J. Q. Wu, M. Seay, V. P. Schulz et al., "Tcf7 is an important regulator of the switch of self-renewal and differentiation in a multipotential hematopoietic cell line," PLoS Genetics, vol. 8, no. 3, article e1002565, 2012.

[19] C. Hernandez-Sanchez, O. Bartulos, and F. de Pablo, "Proinsulin: much more than a hormone precursor in development," Reviews in Endocrine \& Metabolic Disorders, vol. 6, no. 3, pp. 211-216, 2005.

[20] C. Hernandez-Sanchez, A. Mansilla, E. J. de la Rosa, and F. de Pablo, "Proinsulin in development: new roles for an ancient prohormone," Diabetologia, vol. 49, no. 6, pp. 1142-1150, 2006.

[21] A. I. Valenciano, S. Corrochano, F. de Pablo, P. de la Villa, and E. J. de la Rosa, "Proinsulin/insulin is synthesized locally and prevents caspase- and cathepsin-mediated cell death in the embryonic mouse retina," Journal of Neurochemistry, vol. 99, no. 2, pp. 524-536, 2006.

[22] C. Drevon and T. Jaffredo, "Cell interactions and cell signaling during hematopoietic development," Experimental Cell Research, vol. 329, no. 2, pp. 200-206, 2014.

[23] J. A. McCubrey, L. S. Steelman, F. E. Bertrand et al., "Multifaceted roles of GSK-3 and Wnt/beta-catenin in hematopoiesis and leukemogenesis: opportunities for therapeutic intervention," Leukemia, vol. 28, no. 1, pp. 15-33, 2014.

[24] S. Corrochano, R. Barhoum, P. Boya et al., "Attenuation of vision loss and delay in apoptosis of photoreceptors induced by proinsulin in a mouse model of retinitis pigmentosa," Investigative Ophthalmology \& Visual Science, vol. 49, no. 9, pp. 4188-4194, 2008.

[25] R. Shah, R. A. Sidner, M. R. Bochan, and R. M. Jindal, "Reversal of diabetes in streptozotocin-treated rats by intramuscular injection of recombinant adeno-associated virus containing rat preproinsulin II gene," Transplantation Proceedings, vol. 31, no. 1-2, pp. 641-642, 1999. 


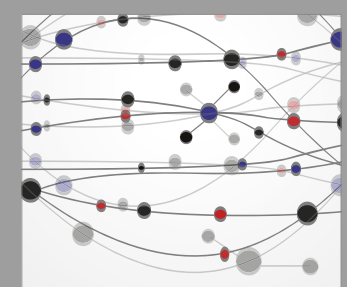

The Scientific World Journal
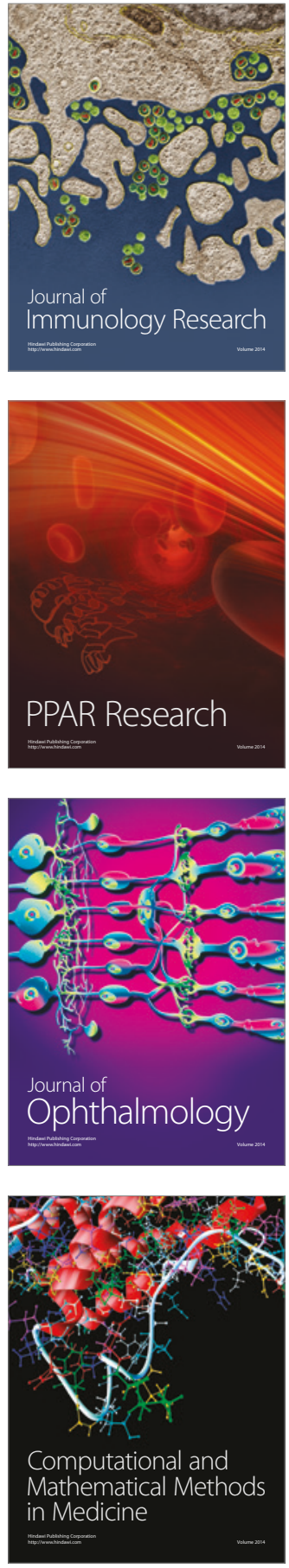

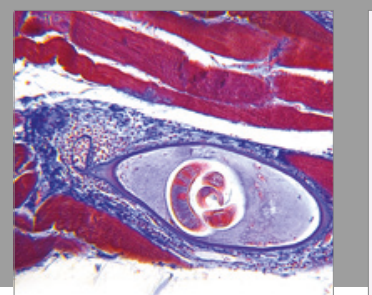

Gastroenterology Research and Practice
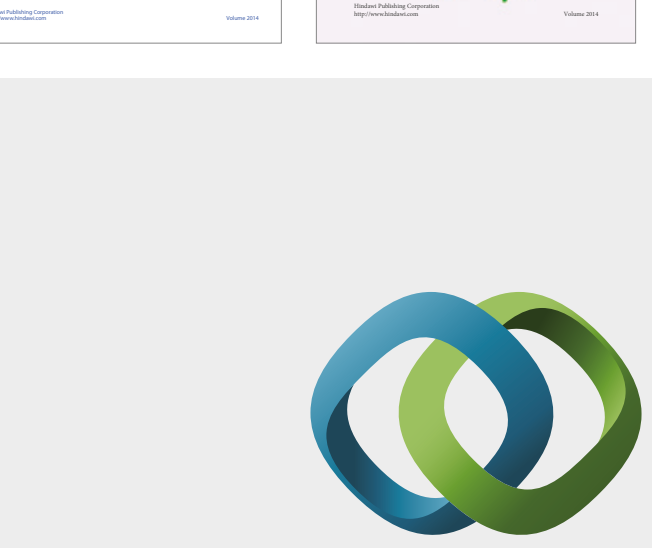

\section{Hindawi}

Submit your manuscripts at

https://www.hindawi.com
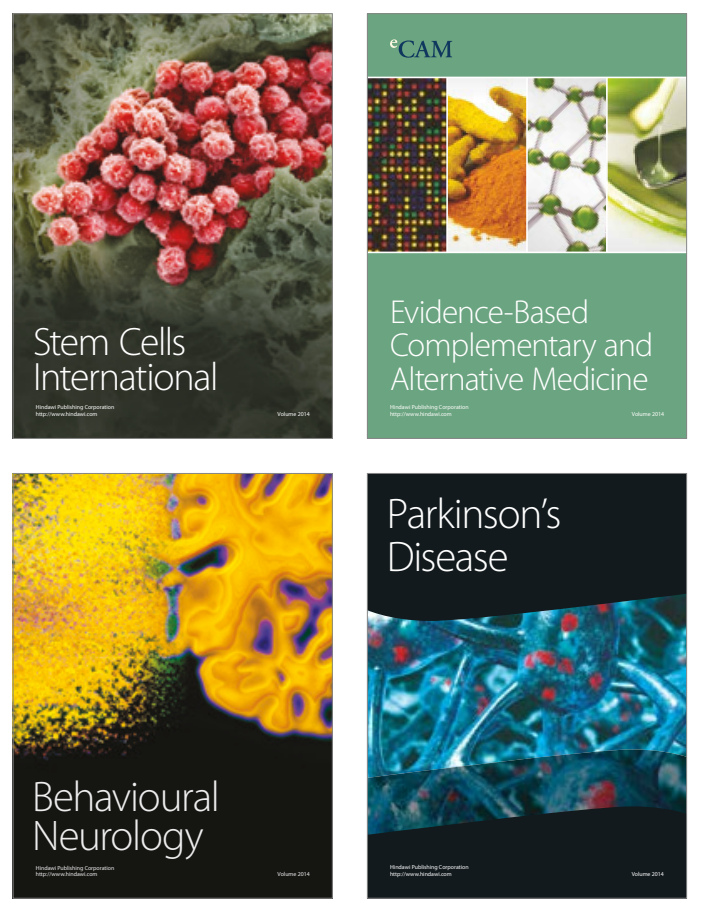
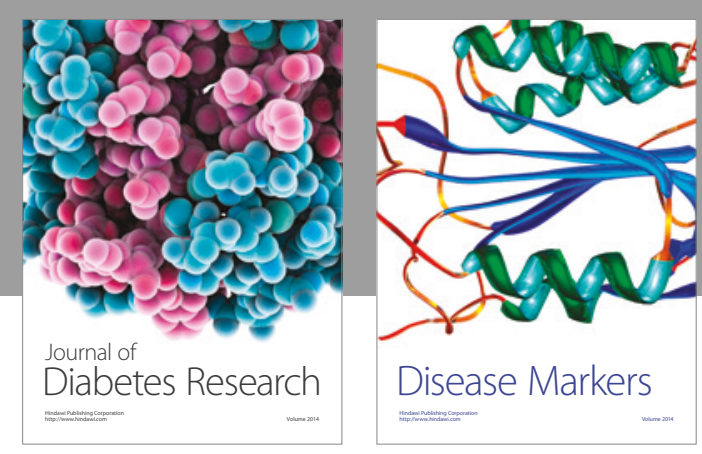

Disease Markers
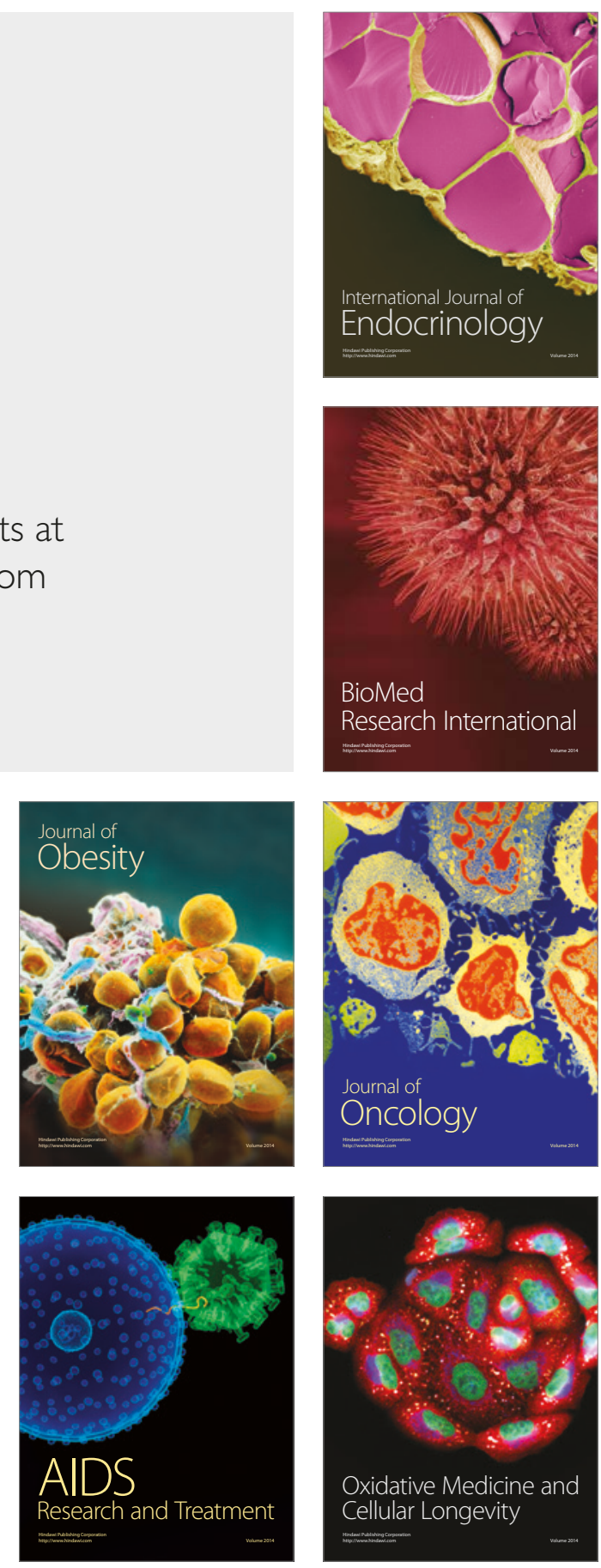